\title{
BMJ Open Incidence of neuromyelitis optica spectrum disorders in China: a large cohort study using claim data
}

\author{
Yiqun Wu (D) , ${ }^{1}$ Mo Yang, ${ }^{2,3}$ Pei Gao (D) , ${ }^{1}$ Zijing Wang, ${ }^{1}$ Junhui Wu, ${ }^{1}$ Jiating Wang, ${ }^{1}$ \\ Quangang $\mathrm{Xu},{ }^{2}$ Huanfen Zhou, ${ }^{2}$ Tao Wu, ${ }^{1}$ Weiping Wu, ${ }^{2}$ Shihui Wei, ${ }^{2}$ \\ Yong-Hua $\mathrm{Hu}$ (i) ${ }^{1}$
}

To cite: Wu Y, Yang M, Gao P, et al. Incidence of neuromyelitis optica spectrum disorders in China: a large cohort study using claim data. BMJ Open 2022;12:e048942. doi:10.1136/ bmjopen-2021-048942

- Prepublication history and additional supplemental material for this paper are available online. To view these files, please visit the journal online (http://dx.doi.org/10.1136/ bmjopen-2021-048942).

YW and MY contributed equally.

Received 26 August 2021 Accepted 13 January 2022

Check for updates

(C) Author(s) (or their employer(s)) 2022. Re-use permitted under CC BY-NC. No commercial re-use. See rights and permissions. Published by BMJ.

${ }^{1}$ Department of Epidemiology and Biostatistics, School of

Public Health, Peking University Health Science Center, Beijing, China

${ }^{2}$ Department of Ophthalmology, The First Medical Center, Chinese PLA General Hospital, Beijing, China

${ }^{3}$ Department of Neuroophthalmology, Eye Hospital, China Academy of Chinese Medical Sciences, Beijing, China

Correspondence to Professor Yong-Hua Hu; yhhu@bjmu.edu.cn and Professor Shihui Wei; weishihui706@hotmail.com

\section{ABSTRACT}

Objectives Population-based studies estimating the incidence of neuromyelitis optica spectrum disorders (NMOSDs) in Asia are limited, and the relationship between latitude and incidence has been scarcely investigated. We aimed to estimate the incidence of NMOSDs in Chinese adults and explore their relationship to latitude.

Design Cohort study based on data from the Urban

Employee Basic Medical Insurance in China.

Participants 177 million people were followed from 2016 to 2017 in 20 provinces.

Primary outcome measures The incidence rate was estimated by Poisson distribution and reported as ageadjusted and sex-adjusted rates using the standard population.

Results There were 1313 incident NMOSD cases, with an overall incidence of 0.41 (95\% Cls: 0.39 to 0.43 ) per 100000 person-years. The incidence in females was higher, with a female-to-male IRR of 4.52 . The incidence increased with age, peaking at 55-64 years in females and 65-74 years in males and then decreasing thereafter. The female-to-male IRRs were higher in those $<55$ years. The association between latitude and incidence was not statistically significant.

Conclusions The incidence of NMOSD in Chinese adults was 0.41 per 100000 person-years. There is no latitude gradient observed. Sex and age influence the risk of NMOSD, suggesting the role of genetic, hormonal and other related factors in the pathophysiology.

\section{INTRODUCTION}

Neuromyelitis optica spectrum disorder (NMOSD) is considered a rare antibodymediated autoimmune central nervous system disorder associated with poor prognosis and a high risk of relapse. ${ }^{1}$ Aquaporin-4 immunoglobulin G (AQP4-IgG) is highly specific for clinically diagnosed NMO and targets a component of the dystroglycan protein complex located in astrocytic foot processes at the blood-brain barrier. ${ }^{2}$ NMOSD occurs in individuals of all ethnicities around the world, with a reported prevalence of 0.7 to 10 per $100000,{ }^{3-12}$ and the incidence is estimated to be $0.037-0.73$ per 100000 person-years. ${ }^{11-14}$
Strengths and limitations of this study

- Based on a relatively large sample, the study estimated the incidence of neuromyelitis optica spectrum disorders in Chinese adults with a homogenous genetic background.

- The incidence between sexes in different age strata was compared and the latitude gradients were explored.

- The separate incidences for seronegative and seropositive cases cannot be estimated because of limited data.

- Increasing the observational period could result in more exact estimates.

It has been reported that NMOSD is more common in the Asian population than in the Caucasian population, ${ }^{11} 121516$ which reflects distinct risks among ethnicities. Although a growing number of epidemiologic studies on NMOSD have been reported in recent years, most of them were conducted in Caucasians. ${ }^{17}$ In Asia, population-based epidemiological studies, especially studies exploring incidence estimates for NMOSD, are still limited. 5781618

A latitude gradient for multiple sclerosis (MS) has been widely reported in previous epidemiological studies, possibly related to low sun exposure and low serum levels of vitamin $\mathrm{D}$, resulting in an immunomodulatory effect. ${ }^{19}$ NMOSD is an autoimmune disease that was once considered a variant of MS, and the risk of NMOSD may also be related to latitude. Because of limited sample sizes due to the low incidence rate, to our knowledge, only three recent studies have tried to explore the latitude gradient in the risk of NMOSD. ${ }^{81118}$ China, with a vast territory, is an ideal setting to study potential differences in the incidence of NMOSD associated with differences in latitude. Hence, in this study, we aimed to estimate the incidence of 
NMOSD in the Chinese population based on a large data source, describe the sex-specific and age-specific aspects, and further investigate the relationship between the incidence and latitude, providing information regarding the epidemiology of NMOSD in Asia and helping to create insights into the susceptibility and pathophysiology of NMOSD.

\section{METHODS}

\section{Study design and data source}

This study was conducted in China. The incidence of NMOSD was estimated based on a retrospective cohort design using a nationwide medical insurance claim database referred to as Urban Employee Basic Medical Insurance (UEBMI).

The UEBMI is one of three basic medical insurance programmes in China. All current and retired employees in government, state-owned enterprises, non-government organisations and private entities are obligated to enrol in UEBMI. Accordingly, the UEBMI database keeps track of all the medical claim records, including inpatients and outpatients, for the UEBMI beneficiaries. According to the data from the Ministry of Human Resources and Social Security in China, about 295 million adults enrolled in the UEBMI programme, accounting for more than one-fifth of the total Chinese population. ${ }^{20}$ For the UEBMI participants, every diagnosis that occurs in all the ranked hospitals, including the primary, secondary and tertiary hospitals, is recorded. As all the hospitals are centralised to the UEBMI system, for the UEBMI database, a diagnosis is recorded once an enrollee is diagnosed with a disease, irrespective of the physical position of diagnosing and treatment, which makes the UEBMI database containing the most complete medical records for the population. The details of the UEBMI database are described elsewhere, and it is useful for epidemiological studies. ${ }^{21}$ Generally, the UEBMI database is a centralised health information system that records each billable medical service, including information on disease diagnosis and the cost of treatment. The database is standardised under the regulation of the National Healthcare Security Administration of China according to the National Health Statistics Management Rule. ${ }^{22}$

The data from 1 January 2013 to 31 December 2017 were extracted and used in this study. Eleven provinces (Jilin, Hebei, Tianjin, Beijing, Ningxia, Tibet, Shaanxi, Guizhou, Jiangxi, Fujian and Shanghai) were excluded due to the absence of diagnosis information or reporting policy exemptions. Ultimately, anonymised patient-level data of 20 provinces were used. Based on the database, a retrospective cohort including 177 million employees followed from 2016 to 2017 was created, with large coverage of the whole UEBMI population. The mean age of the cohort population is 42.8 years and $52 \%$ are males.

\section{Case definition}

The diagnosis for NMOSD in hospitals was according to the 2015 International Panel for Neuromyelitis Optica Diagnosis criteria. ${ }^{1}$ AQP4 antibody test and MRI were detected when necessary. The incident cases were identified as the patients with newly diagnosed NMOSD in 2016 and 2017 according to the following criteria: (1) diagnosed according to the 10th revision of the International Statistical Classification of Diseases and Related Health Problems G36.001/G36.951 codes or diagnosed with 'neuromyelitis optica', 'NMO', 'neuromyelitis optica spectrum disorder', 'NMOSD' or 'Devic's disease'; (2) diagnosed between 1 January 2016 and 31 December 2017; (3) no diagnoses of NMO or NMOSD from 1 January 2013 to 31 December 2015, which means the dataset between 2013 and 2015 was used as the wash-out period; and (4) complete information on birth date, sex and race. In this study, only $0.03 \%$ of the records were deleted because of incomplete information. A total of 1813 NMOSD cases in 2016 and 2017 were identified, with 1313 newly diagnosed cases and 700 previously diagnosed cases between 2013 and 2015 . There were $84.1 \%$ (1104 cases) and $15.9 \%$ (209 cases) of the newly diagnosed cases treated as inpatients and outpatients, respectively.

\section{Estimation of incidence}

The population at risk for the incidence estimates was determined by the number of UEBMI beneficiaries from 2016 to 2017 (approximately 177 million). Because of the extremely low incidence of NMOSD, the pre-existing cases $(\mathrm{n}=700)$ of NMOSD in the 3-year wash-out period (2013-2015) were not excluded from the population at risk. The incidence rate was calculated with the number of newly diagnosed NMOSD cases between 2016 and 2017 as the numerator and the number of person-years at risk from 2016 through 2017 as the denominator. Age-specific and sex-specific incidence rates were also calculated. As the cohort in our study lived in 20 provinces located from $20^{\circ}$ to $46^{\circ}$ north of the Earth's equatorial plane, it provided an excellent chance to explore the latitude distribution of the incidence of NMOSD. Therefore, the whole cohort was divided into five subgroups according to their latitude of residence $\left(20^{\circ}, 25^{\circ}, 30^{\circ}, 35^{\circ}\right.$ and $\left.40^{\circ}\right)$, and the incidence rates in each subgroup were calculated.

\section{Statistical analysis}

The incidence and the 95\% CIs were estimated using a Poisson distribution. The age-adjusted and sex-adjusted rates were reported using residents in the sixth demographic census in 2010 in China as the standard population. The incidence rates were calculated and described as the number of incident cases per 100000 person-years. In addition, the total population was stratified by race, age, sex and latitude, and incidence rates were calculated in each stratified group separately. The incidence rate ratio (IRR) and the 95\% CIs were also reported. Estimations among only the Chinese Han people were also performed to reflect a more homogeneous population. Quantitative 
Table 1 Incidence of NMOSD in China, 2016-2017

\begin{tabular}{|c|c|c|c|c|}
\hline & Number of cases & Person-years & $\begin{array}{l}\text { Incidence } \\
\text { (95\% CI, per } 100000 \\
\text { person-years) }\end{array}$ & IRR (95\% Cl) \\
\hline Overall & 1313 & 348909063 & 0.41 (0.39 to 0.43$)$ & - \\
\hline \multicolumn{5}{|l|}{ Race } \\
\hline Han & 1133 & 304312130 & 0.40 (0.38 to 0.42$)$ & 0.83 (0.73 to 0.96$)$ \\
\hline Female & 1031 & 156344490 & 0.68 (0.63 to 0.72$)$ & 4.52 (4.39 to 4.65$)$ \\
\hline Male & 282 & 192564573 & 0.15 (0.13 to 0.17$)$ & Ref \\
\hline \multicolumn{5}{|l|}{ Age, years } \\
\hline $18-34$ & 260 & 129539892 & 0.21 (0.19 to 0.24$)$ & Ref \\
\hline $65-74$ & 114 & 20836156 & 0.61 (0.50 to 0.73 ) & 2.89 (2.66 to 3.11$)$ \\
\hline $75+$ & 44 & 14208486 & 0.37 (0.26 to 0.50$)$ & 1.73 (1.40 to 2.06$)$ \\
\hline
\end{tabular}

IRR, incidence rate ratio; NMOSD, neuromyelitis optica spectrum disorder.

variables are described as the mean $\pm \mathrm{SD}$, and categorical variables are described as numbers (percentages). Pearson's $\chi^{2}$ test was used to test the differences in incidences between groups. The Pearson correlation coefficient was calculated to measure the linear correlation between the incidence of NMOSD in different provinces and their latitudes. The $\chi^{2}$ test for trend in proportion was further used to test the trend of incidences in five latitudinal groups. Values of $p<0.05$ were considered statistically significant. The median annual medical cost for each patient was also presented. Analyses were performed using R (V.3.6.0). The Chinese and world map data were obtained using package 'maptools' and 'rnaturalearth' in R, respectively, and all the figures were plotted by package 'ggplot2'.

Patient and public involvement

No patient involved.

\section{RESULTS}

Among 177229403 UEBMI beneficiaries, 1313 newly diagnosed NMOSD cases were identified from 2016 to 2017, with 348909063 observed person-years (table 1). Most of the patients were Han Chinese, accounting for $86.2 \%$ of the patients $(n=1133)$. Most of the patients were female $(\mathrm{n}=1031,78.5 \%)$. The mean age at onset was 47.6 years (19 years- 89 years, SD: 13.7 years). The mean onset age among female patients (mean: 47.0 years, SD: 13.4 years) was younger than that among male patients (mean: 49.7 years, SD: 14.7 years), and the differences between the two sexes were statistically significant $(\mathrm{p}=0.004)$.

The overall incidence rate was 0.41 (95\% CI: 0.39 to 0.43) per 100000 person-years (table 1 ). The incidence in the Han population was lower than that in other populations (IRR: $0.83,95 \%$ CI: $0.73,0.96$, table 1 ).

The incidence rate in females was significantly higher than that in males (females: $0.68,95 \%$ CI: 0.63 to 0.72 ; males: $0.15,95 \%$ CI: 0.13 to 0.17 , per 100000 personyears; $\mathrm{p}<0.001)$, with a female-to-male IRR of $4.52(95 \%$ CI: 4.39 to 4.65$)$. When including only the Han race, the incidence rate in females was 0.66 (95\% CI: 0.62 to 0.70 ) per 100000 person-years, which was also significantly higher than that in males $(0.15,95 \%$ CI: 0.13 to 0.17 , per 100000 person-years, $\mathrm{p}<0.001$ ) (online supplemental table 1).

The incidence rates in different age groups are shown in table 1 . The incidence rate was the lowest in those aged between 18 and 34 years (0.21, 95\% CI: 0.19 to 0.24 , per 100000 person-years). As age increased, the incidence rate increased, with the highest rate in those between 55 and 64 years old $(0.67,95 \%$ CI: 0.58 to 0.76 , per 100000 person-years), and then the incidence decreased in older groups (the solid line in figure 1, online supplemental table 1). Similar patterns were seen for age-specific incidences in both sexes (dash-dotted and dotted lines in figure 1, online supplemental table 1). However, the highest rate in females was observed in the group aged between 55 and 64 years $(1.09,95 \%$ CI: 0.93 to 1.26 , per 100000 person-years), while the highest rate among males was in the group aged between 65 and 74 years $(0.28,95 \%$ CI: 0.20 to 0.39 , per 100000 person-years). The femaleto-male IRRs were 4.68 (95\% CI: 4.38 to 4.99 ), 5.05 (95\% CI: 4.75 to 5.35$), 5.24$ (95\% CI: 4.98 to 5.49$), 4.26$ (95\% CI: 3.96 to 4.56 ), 3.34 (95\% CI: 2.94 to 3.74 ) and 3.02 (95\% CI: 2.40 to 3.64) for those aged 18-34, 35-44, 


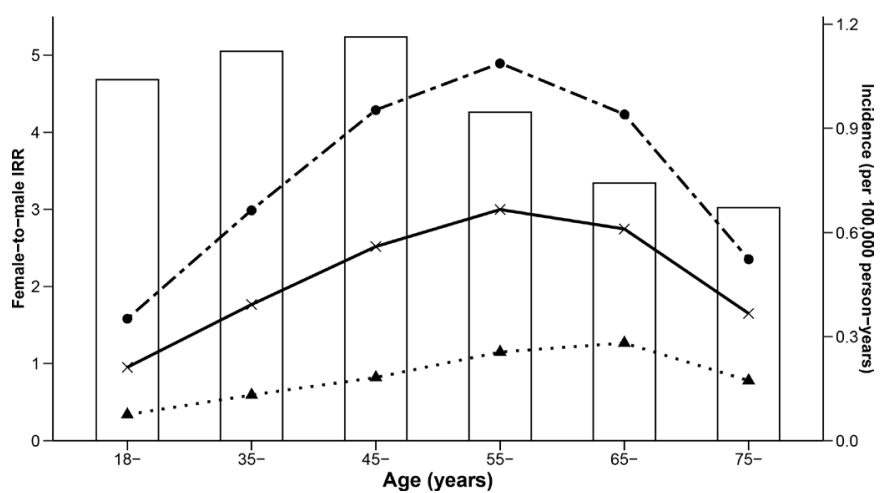

Figure 1 Age-specific and sex-specific incidence and female-to-male IRR in different age groups. Bar: IRRs of females to males; lines: incidence rate; dash-dotted line: incidence rate in females; dotted line: incidence rate in males; solid line: incidence rate in overall residents. IRR, incidence rate ratio.

45-54, 55-64, 64-74 and over 75 years, respectively; these values were higher in individuals younger than 55 years old (bars in figure 1, online supplemental table 1).
When including only the Han participants, the incidence rates in different age groups ranged from 0.20 to 0.60 per 100000 person-years, with female-to-male IRRs ranging from 3.05 to 5.20 , and the highest incidence rates in females and males were still in the 55-64 and 65-74 year age groups, respectively (online supplemental table 1).

No significant correlation was detected between the incidence rates in cities in 20 provinces and latitude $(\mathrm{R}=0.03, \mathrm{p}=0.896$, figure 2$)$. The incidence rates in cities located $20^{\circ}, 25^{\circ}, 30^{\circ}, 35^{\circ}$ and $40^{\circ}$ north of Earth's equatorial plane were 0.53 (95\% CI: 0.46 to 0.61$), 0.41(95 \%$ CI: 0.33 to 0.50$), 0.40$ (95\% CI: 0.36 to 0.43$), 0.54(95 \%$ CI: 0.46 to 0.62 ) and 0.30 (95\% CI: 0.26 to 0.35 ) per 100000 person-years, respectively. There were no significant trends in incidence rates according to latitude in the total sample (figure $3, P$-trend=0.874) or in the two sex subgroups (online supplemental table 2) (female: $P$ trend $=0.825$, male: $P$-trend $=0.953$ ). When including only Han individuals, the results did not change substantially (online supplemental table 2 and figure 1).

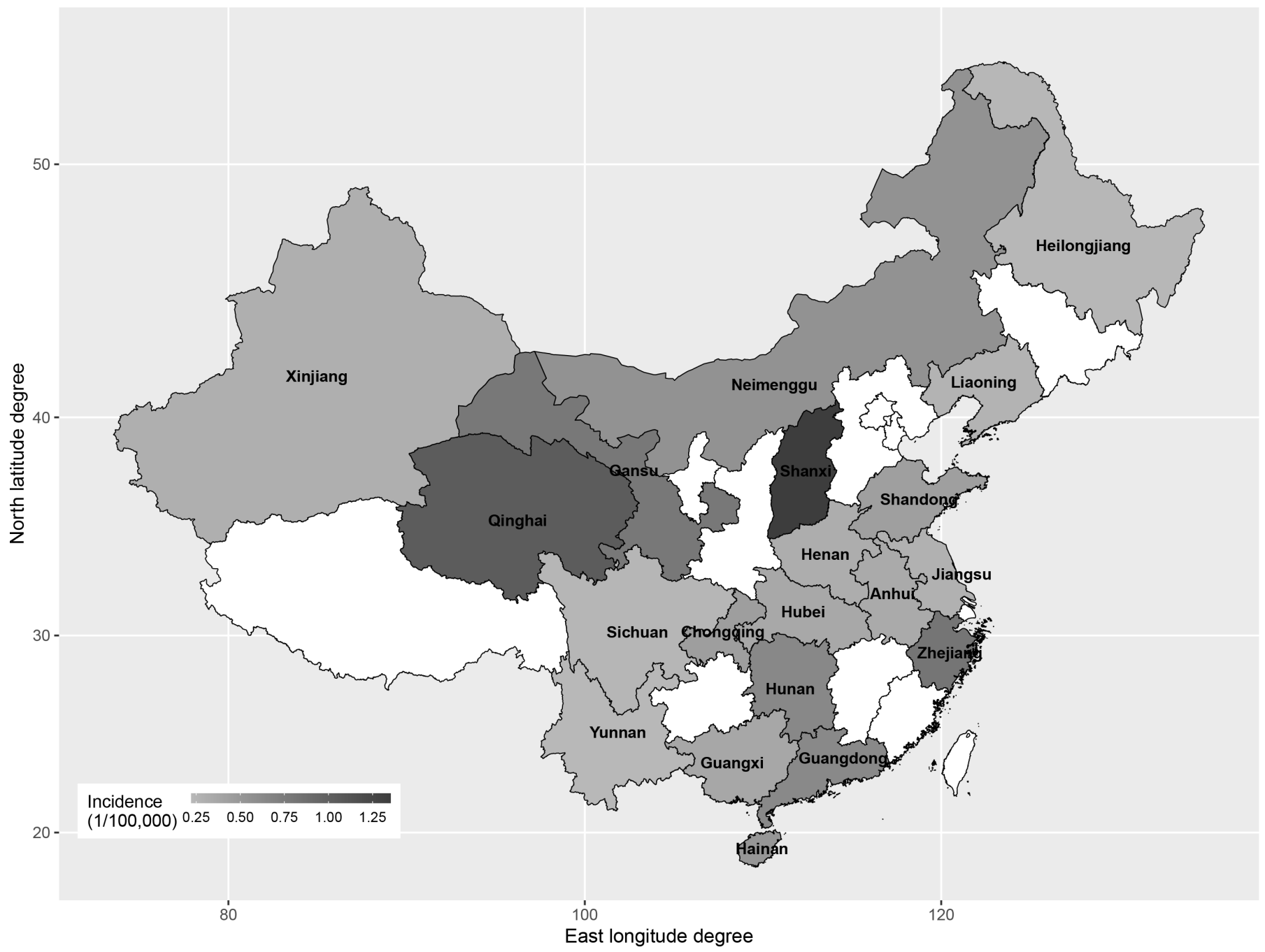

Figure 2 Incidence rates of neuromyelitis optica spectrum disorder in 20 provinces in China. The geospatial vector data were downloaded from http://wwwdiva-gisorg/gdata. The shapefiles were read using the 'maptools' package first, then the map was drawn using the 'ggplot2' package in R (V.3.6.0). 


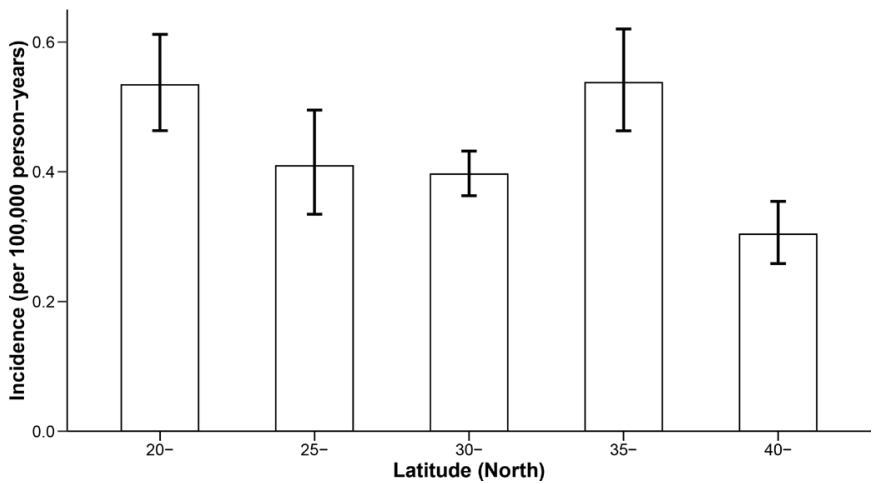

Figure 3 Incidence rates of NMOSD in areas with different latitudes. The bars show the incidence rate estimates in each latitude subgroup, and the vertical lines show the $95 \% \mathrm{Cls}$ of incidence rates. NMOSD, neuromyelitis optica spectrum disorder.

For each patient, the median annual total medical cost was 13225 RMB Yuan, most of which was reimbursed by the UEBMI programme remaining a median annual cost of $2787 \mathrm{RMB}$ Yuan paid by each patient.

\section{DISCUSSION}

Based on a large cohort including 177 million people followed for 2 years, we identified 1313 newly diagnosed NMOSD cases, and the incidence of NMOSD in Chinese adults was estimated as 0.41 per 100000 person-years. Although the literature indicated that NMOSD is more common in the Asian population, there was only one recent epidemiological study (Tian et al 2020) ${ }^{18}$ in China presenting an incidence of 0.347 per 100000 person-years in adults. Despite differences that exist in study design, our study and Tian's study reached similar conclusions. Under the supervision of the Chinese National Health Commission, the diagnostic procedure on NMOSD was the same across hospitals. The newly diagnosed NMOSD cases partially overlap between Tian's study and our study, that Tian's study included inpatient records of residents from all tertiary hospitals in 31 provinces, while this study included inpatient and outpatient records of UEBMI beneficiaries from all ranked hospitals in 20 provinces. According to our data, most newly diagnosed patients with NMOSD were treated as inpatients (1104 cases, 84.1\%). Including records of outpatients (209 cases, 15.9\%) may partially explain the slightly higher incidence estimation in our study.

Most previous studies investigating the incidence rate of NMOSD were conducted among Caucasians. ${ }^{349-11} 1323$ As table 2 and online supplemental figure 2 show, the incidence rates (per 100000 person-years) of NMOSD were reported to be 0.037 in Australia and New Zealand, ${ }^{11} 0.063$ in Catalonia (Spain), ${ }^{4} 0.07$ in Olmsted County (USA), ${ }^{23}$ 0.07 in Denmark ${ }^{10}$ and 0.132 in Hungary, ${ }^{9}$ which were much lower than the incidence reported in our study. However, the incidence (per 100000 person-years) was 0.73 in Martinique $(90 \% \text { of the population was black })^{23}$ and 0.73 in Korea; ${ }^{7}$ which were higher than the incidence in our results. Although differences in the study designs may contribute partly, the distinct results between studies may mostly be due to interethnic differences in the risk of NMOSD, such as the differences in genetic background, infectious exposure, and other risk factors. ${ }^{7}$

Similar to other reports (table 2), the predominance in females was also observed in our study. The incidence rate (per 100000 person-years) was 0.68 in females compared with 0.15 in males. The female-to-male ratios varied in previous reports, 46710162324 with the highest ratio observed in Malaysia (10.6), ${ }^{16}$ and the lowest ratio observed in Mangalore, South India (1.2). ${ }^{24}$ The femaleto-male ratios reported in most previous studies were calculated by the number of cases. In our study, we reported a female-to-male IRR of 4.52 (95\% CI: 4.39 to 4.65), which compared the risks between the two sexes based on standard time frames and allowed for a better understanding of the relative risks of NMOSD between sexes. The reasons for sex differences in NMOSD may be similar to the reasons for sex differences in other autoimmune diseases, as there was a general female predominance in autoimmune diseases, such as systemic lupus erythematosus, MS and autoimmune thyroid disease, though there are exceptions. ${ }^{25}$ Immunocompetence and immune reactivity differ according to sexes, and hormonal factors, genetic susceptibility, epigenetics and environmental factors have been suggested to be involved. ${ }^{25} 26$ Recent studies also reported different clinical features ${ }^{27}$ and outcomes ${ }^{28}$ of NMOSD between sexes. Therefore, in studies concentrating on mechanisms of NMOSD, sex should be a main focus.

Due to a limited number of cases, few studies have assessed the incidence of NMOSD by age strata. Based on more than 1000 NMOSD cases, our results showed that the incidence increased with increasing age, with a peak in the 55-64-year-old group, and decreased thereafter. This was similar to a report from Japan. ${ }^{8}$ Interestingly, our results showed that this increase-then-decrease pattern was more pronounced in females, with a peak incidence in the 55-64 age group, while the incidence in males increased modestly until individuals reach an age between 65 and 74 , which led to the highest female-to-male IRR among individuals 45-54 years old and a sustained decrease in IRR thereafter. Similarly, a recent study reported that the female-to-male ratio was pronounced at reproductive ages. ${ }^{29}$ For females, substantial hormone changes occur during the menopausal period, ${ }^{30}$ which may influence the immune status and increase the susceptibility to autoimmune disease. ${ }^{3132}$ The hormonal factors together with other genetic and environmental factors may contribute to the higher level of female-to-male IRRs for NMOSD in individuals under 55 years old.

Vitamin D is involved in nearly all important biological functions related to autoimmune demyelinating disease. ${ }^{33}$ A high prevalence of vitamin D deficiency among patients with NMOSD and MS has been reported. ${ }^{19}$ As sun avoidance is one of the factors related to vitamin D 

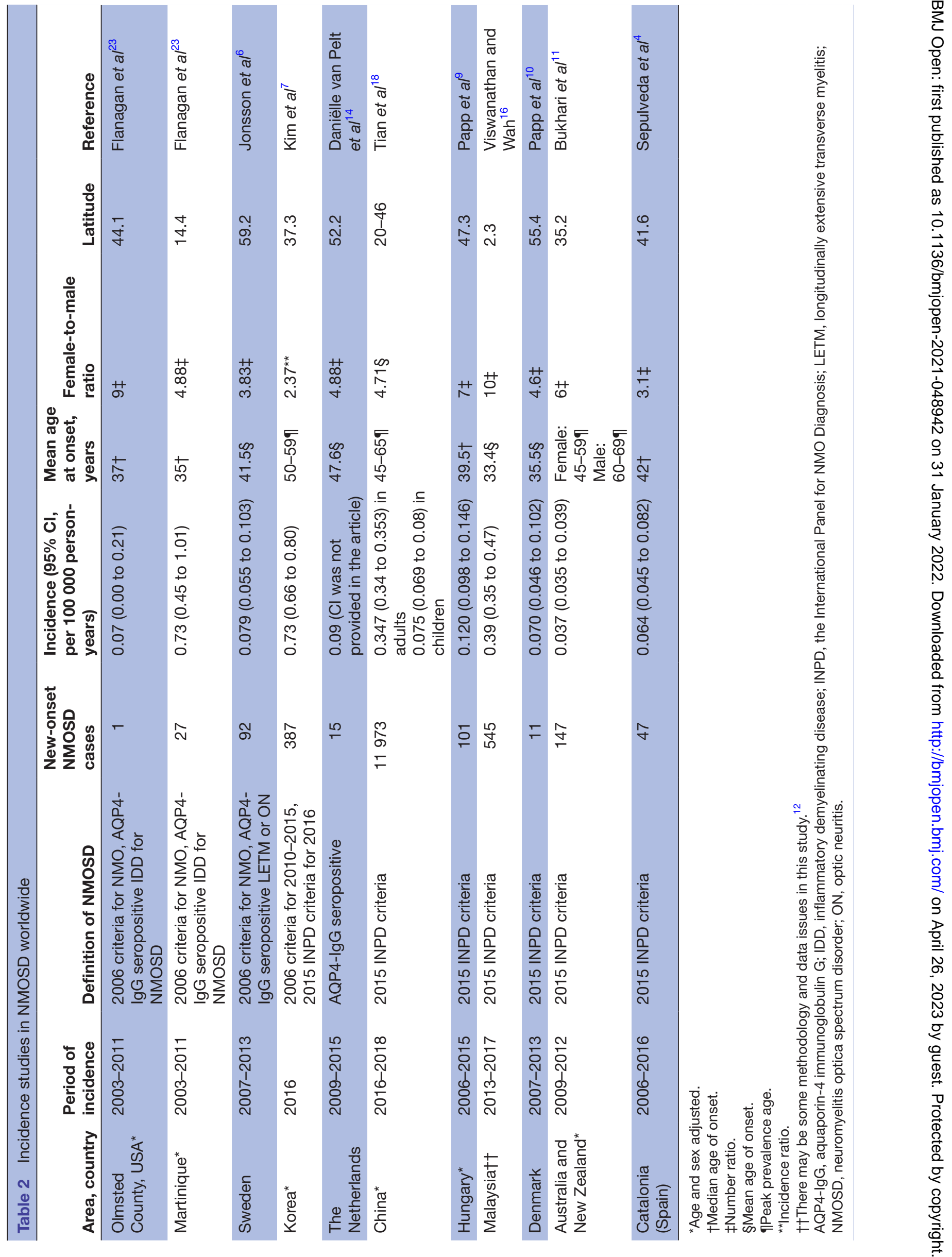
deficiency, robust latitudinal gradients in prevalence ${ }^{34}$ and incidence ${ }^{35}$ for MS have been reported, with higher risks associated with higher latitudes. As an autoimmune disease once considered a variant of MS, NMOSD was hypothesised to exhibit a latitude gradient similar to that associated with MS; however, mainly due to limited cases of this rare disease, epidemiological studies reporting latitudinal gradients in NMOSD are limited, and whether there is an association between latitude and the risk of NMOSD is still unclear. One study reported a higher prevalence of NMOSD in Southern Japan than in Northern Japan, ${ }^{8}$ and another study reported a reverse relationship between latitude and prevalence in Australia and New Zealand. ${ }^{11}$ In our study, the cohort was enrolled from 20 provinces located from $20^{\circ}$ to $46^{\circ}$ latitude, providing an opportunity to test the hypothesis, but the results did not show significant associations between latitude and the incidence of NMOSD. This result was similar to Tian et $a l$ s study. ${ }^{18}$ According to the results (figure 3), there may be trends towards lower incidence at higher latitudes, except for the high incidence from $35^{\circ}$ to $40^{\circ}$. The high incidences in this region were mainly attributed to three provinces (Qinghai, Gansu and Shanxi, figure 2). A sightly higher percentage of UEBMI beneficiaries were minority races in the three provinces $(18.6 \%$ vs $12.6 \%)$. According to our results, the incidence in minority races were higher than that in Han people ( 0.48 vs 0.40 per 100000 person-years). However, in the sensitivity analysis when only keeping Han people, a similar latitude pattern (online supplemental table 2) and similar high incidences in the three provinces were observed (online supplemental figure 1). The number of hospitals or neurologists per capita in these three provinces is consistent with the average in China. In Tian's study, similar higher incidences were observed in Qinghai and Shanxi provinces. ${ }^{18}$ We do not have a clear answer to explain the high incidences there. As small increases in the number of incident cases will cause significant changes in the rate of a rare disease, the results need to be verified with a longer observational period with larger numerators. We further labelled the latitude degree for the study regions in previous studies (table 2, online supplemental figure 2), but still, no latitude gradients were shown among studies. Nevertheless, we cannot exclude the possibility of latitude gradients in the risk of NMOSD. Our study may be underpowered, and the heterogeneity between studies led to uncertainty. Broad collaborations are needed to further explore latitude gradients for a better understanding of NMOSD. On the other hand, the latitude gradient was assumed to be related to vitamin $\mathrm{D}$ deficiency and then associated with the risk of MS. Vitamin D may be involved in different biological mechanisms in NMOSD than in MS, which also needs further exploration.

There were limitations in this study. First, because of limited data availability, we could not obtain information on the antibody status to estimate the incidences for seronegative and seropositive cases separately. Further studies are needed to explore the age, sex and latitude distributions for different subclinical types. Second, the 2-year time frame in this study was chosen to optimise the number of cases, and commercial assays for AQP4-IgG have been widely used since 2015 in most areas of China. There is a possibility of underdiagnosis before 2015. As the incident cases in the study were newly diagnosed cases other than new-onset cases, the uneven availability of the AQP4 antibody test during the wash-out period may lead to an overestimation of the incidence. Increasing the observational period could result in more exact estimates. Third, the latitude analysed in this study reflected the locations where the person enrolled in the UEBMI programme. For the majority of UEBMI beneficiaries, the places where they enrolled in the UEBMI programme are the same as the locations they are working and living. However, we cannot exclude the possibility that the location where a patient developed NMOSD was different from his address. Forth, the study population was composed of employees enrolled in UEBMI who may have different characteristics from those not included. Also, data from 11 provinces were not included. We found similar age and sex distribution between the excluded and included provinces, but a higher gross domestic product in the included provinces (online supplemental table 3). The extrapolation of the results should be done with caution. Despite these limitations, in this study, most people in the cohort were Chinese Han, providing a homogenous genetic background in the estimations. The relatively large sample size makes it possible to compare the incidence between sexes, estimate the female-to-male IRRs in different age strata and explore the latitude gradients. The results were helpful in providing insights into the pathophysiology of NMOSD.

In conclusion, the incidence of NMOSD in the Chinese adults was 0.41 per 100000 person-years, which is higher than that among Caucasians. The incidence of NMOSD varies by age and sex strata. Genetic, hormonal and other related factors may play roles in the pathophysiology of NMOSD. There is no latitude gradient observed. The results provide information on the epidemiological profiles of NMOSD in Asia and help to provide insights into NMOSD susceptibility to better understand the condition.

Contributors Y-HH and SW contributed to the study concept, had full access to all the data, and acted as guarantors. YW and MY take responsibility for the integrity of the data and interpreted the findings and drafted the article. PG, TW, ZW, JWu and JWa contributed to data analysis. PG, TW, WW, QX and HZ interpreted the data. All the authors contributed to the critical revision of the article for important intellectual content. The corresponding author attests that all listed authors meet authorship criteria and that others not meeting the criteria have been omitted.

Funding The authors have not declared a specific grant for this research from any funding agency in the public, commercial or not-for-profit sectors.

Map disclaimer The inclusion of any map (including the depiction of any boundaries therein), or of any geographic or locational reference, does not imply the expression of any opinion whatsoever on the part of BMJ concerning the legal status of any country, territory, jurisdiction or area or of its authorities. Any such expression remains solely that of the relevant source and is not endorsed by BMJ. Maps are provided without any warranty of any kind, either express or implied.

Competing interests None declared. 
Patient consent for publication Not applicable.

Ethics approval As the data used for this study were collected for administrative purposes without any individual identifiers, this study was exempted from the requirement of Institutional Review Board approval by the Ethics Committee of Peking University Health Science Center, Beijing, China.

Provenance and peer review Not commissioned; externally peer reviewed.

Data availability statement Data are available upon reasonable request. Summarised data can be shared by request from any qualified investigator to the National Insurance Claims for Epidemiological Research (NICER) Group, School of Public Health, Peking University. Contact Email: 0016156078@bjmu.edu.cn.

Supplemental material This content has been supplied by the author(s). It has not been vetted by BMJ Publishing Group Limited (BMJ) and may not have been peer-reviewed. Any opinions or recommendations discussed are solely those of the author(s) and are not endorsed by BMJ. BMJ disclaims all liability and responsibility arising from any reliance placed on the content. Where the content includes any translated material, BMJ does not warrant the accuracy and reliability of the translations (including but not limited to local regulations, clinical guidelines, terminology, drug names and drug dosages), and is not responsible for any error and/or omissions arising from translation and adaptation or otherwise.

Open access This is an open access article distributed in accordance with the Creative Commons Attribution Non Commercial (CC BY-NC 4.0) license, which permits others to distribute, remix, adapt, build upon this work non-commercially, and license their derivative works on different terms, provided the original work is properly cited, appropriate credit is given, any changes made indicated, and the use is non-commercial. See: http://creativecommons.org/licenses/by-nc/4.0/.

\section{ORCID iDs}

Yiqun Wu http://orcid.org/0000-0002-5554-1678

Pei Gao http://orcid.org/0000-0001-8649-1290

Yong-Hua Hu http://orcid.org/0000-0003-1631-3952

\section{REFERENCES}

1 Wingerchuk DM, Banwell B, Bennett JL, et al. International consensus diagnostic criteria for neuromyelitis optica spectrum disorders. Neurology 2015;85:177-89.

2 Lennon VA, Kryzer TJ, Pittock SJ, et al. IgG marker of optic-spinal multiple sclerosis binds to the aquaporin-4 water channel. J Exp Med 2005;202:473-7.

3 Eskandarieh S, Nedjat S, Azimi AR, et al. Neuromyelitis optica spectrum disorders in Iran. Mult Scler Relat Disord 2017;18:209-12.

4 Sepúlveda M, Aldea M, Escudero D, et al. Epidemiology of NMOSD in Catalonia: influence of the new 2015 criteria in incidence and prevalence estimates. Mult Scler 2018;24:1843-51.

5 Houzen $\mathrm{H}$, Kondo K, Niino M, et al. Prevalence and clinical features of neuromyelitis optica spectrum disorders in northern Japan. Neurology 2017;89:1995-2001.

6 Jonsson DI, Sveinsson O, Hakim R, et al. Epidemiology of NMOSD in Sweden from 1987 to 2013: a nationwide population-based study. Neurology 2019;93:e181-9.

$7 \mathrm{Kim}$ JEet al. Prevalence and incidence of neuromyelitis optica spectrum disorder and multiple sclerosis in Korea. Multiple sclerosis 2019:1352458519888609.

8 Miyamoto K, Fujihara K, Kira J-I, et al. Nationwide epidemiological study of neuromyelitis optica in Japan. J Neurol Neurosurg Psychiatry 2018;89:667-8.

9 Papp V, Iljicsov A, Rajda C, et al. A population-based epidemiological study of neuromyelitis optica spectrum disorder in Hungary. Eur $J$ Neurol 2020;27:308-17.

10 Papp V, Illes Z, Magyari M, et al. Nationwide prevalence and incidence study of neuromyelitis optica spectrum disorder in Denmark. Neurology 2018;91:e2265-75.

11 Bukhari W, Prain KM, Waters P, et al. Incidence and prevalence of NMOSD in Australia and New Zealand. J Neurol Neurosurg Psychiatry 2017;88:632-8.
12 Papp V, Magyari M, Aktas O, et al. Worldwide incidence and prevalence of neuromyelitis optica: a systematic review. Neurology 2021;96:59-77.

13 Dale GH, Svendsen KB, Gjelstrup MC, et al. Incidence of neuromyelitis optica spectrum disorder in the central Denmark region. Acta Neurol Scand 2018;137:582-8.

14 Daniëlle van Pelt E, Wong YYM, Ketelslegers IA, et al. Incidence of AQP4-IgG seropositive neuromyelitis optica spectrum disorders in the Netherlands: about one in a million. Mult Scler J Exp Trans/ Clin 2016;2:205521731562565.

15 Ochi H, Fujihara K. Demyelinating diseases in Asia. Curr Opin Neurol 2016;29:222-8

16 Viswanathan S, Wah LM. A nationwide epidemiological study on the prevalence of multiple sclerosis and neuromyelitis optica spectrum disorder with important multi-ethnic differences in Malaysia. Mult Scler 2019;25:1452-61.

17 Etemadifar M, Nasr Z, Khalili B, et al. Epidemiology of neuromyelitis optica in the world: a systematic review and meta-analysis. Mult Scler Int 2015;2015:174720.

18 Tian D-C, Li Z, Yuan M, et al. Incidence of neuromyelitis optica spectrum disorder (NMOSD) in China: a national population-based study. Lancet Reg Health West Pac 2020;2:100021.

19 Rodney C, Rodney S, Millis RM. Vitamin D and demyelinating diseases: neuromyelitis optica (NMO) and multiple sclerosis (MS). Autoimmune Dis 2020;2020:8718736.

20 Ministry of Human Resources and Social Security. Statistical Communiqué on human resources and social security development in 2016. Available: http://www.mohrss.gov.cn/ SYrlzyhshbzb/zwgk/szrs/tjgb/201805/W020180521567132619037. pdf

21 Tian $\mathrm{Y}$, Liu $\mathrm{H}, \mathrm{Wu} \mathrm{Y}$, et al. Association between ambient fine particulate pollution and hospital admissions for cause specific cardiovascular disease: time series study in 184 major Chinese cities. BMJ 2019;367:16572.

22 People's Republic of China Health System Review. Health systems in transition. 5, 2015. ISBN: 9789290617280.

23 Flanagan EP, Cabre P, Weinshenker BG, et al. Epidemiology of aquaporin-4 autoimmunity and neuromyelitis optica spectrum. Ann Neurol 2016;79:775-83

24 Pandit L, Kundapur R. Prevalence and patterns of demyelinating central nervous system disorders in urban Mangalore, South India. Mult Scler 2014;20:1651-3.

25 Ngo ST, Steyn FJ, McCombe PA. Gender differences in autoimmune disease. Front Neuroendocrinol 2014;35:347-69.

26 Voskuhl RR, Gold SM. Sex-related factors in multiple sclerosis susceptibility and progression. Nat Rev Neurol 2012;8:255-63.

27 Pandit L, Asgari N, Apiwattanakul M, et al. Demographic and clinical features of neuromyelitis optica: a review. Mult Scler 2015;21:845-53.

28 Palace J, Lin D-Y, Zeng D, et al. Outcome prediction models in AQP4-IgG positive neuromyelitis optica spectrum disorders. Brain 2019;142:1310-23

29 Borisow N, Kleiter I, Gahlen A, et al. Influence of female sex and fertile age on neuromyelitis optica spectrum disorders. Mult Scler 2017;23:1092-103.

30 Burger HG. The endocrinology of the menopause. Maturitas 1996;23:129-36.

31 Kovats S. Estrogen receptors regulate innate immune cells and signaling pathways. Cell Immunol 2015;294:63-9.

32 Desai MK, Brinton RD. Autoimmune disease in women: endocrine transition and risk across the lifespan. Front Endocrinol 2019;10:265.

33 Spanier JA, Nashold FE, Mayne CG, et al. Vitamin D and estrogen synergy in Vdr-expressing CD4(+) T cells is essential to induce Helios(+)FoxP3(+) T cells and prevent autoimmune demyelinating disease. J Neuroimmunol 2015;286:48-58.

34 Simpson S, Wang W, Otahal P, et al. Latitude continues to be significantly associated with the prevalence of multiple sclerosis: an updated meta-analysis. J Neurol Neurosurg Psychiatry 2019;90:1193-200.

35 Alonso A, Hernán MA. Temporal trends in the incidence of multiple sclerosis: a systematic review. Neurology 2008;71:129-35. 